\title{
Análisis De Los Riesgos Mecánicos Y Su Incidencia En La Seguridad Y Salud Laboral En Los Trabajadores. Estudio De Caso: Fundición De Estructuras Metálicas
}

\author{
Jairón Enrique Mendoza Proaño \\ Magister en Seguridad, Higiene Industrial y Salud Ocupacional \\ Carlos Julio Molestina Malta \\ Ingeniero Industrial \\ Docente en la Universidad de Guayaquil, Ecuador \\ Edison Antonio Chango Agama \\ Sofía Andrea Basantes Vinueza \\ Ingeniera en Ecoturismo
}

doi: 10.19044/esj.2017.v13n15p352 URL:http://dx.doi.org/10.19044/esj.2017.v13n15p352

\begin{abstract}
The analysis of occupational risks within companies, based on civil works, is of great importance in ensuring the safety and the occupational health of its collaborators. The present research work focuses on the analysis of the current situation by means of the methodology of William Fine. The study aims to identify and evaluate the mechanical risks, where the occurrence of these catastrophic events generates multiple consequences. This consequence is both for the employer and the employee, and it ranges from labor absenteeism, physical and mental injuries, and significant economic losses for enterprises by various cases of demands or treatments that must incur due to the lack of procedures of control and prevention. This, however, might be equipment and machinery used in the phase of casting metallic structures, especially in Quintana construction company. The results were obtained through qualitative-quantitative methodology in obtaining the worker's perspectives. They stated that they are conditioned to high risk mechanical jobs, where the lack of equipment for protection as well as the proper socialization of internal safety guidelines limited the involvement of partners. Consequently, it is essential to mitigate the occurrence of risks at the mention of the company events. As part of the conclusion of this study, there is the need to design a matrix of risks and identify the existing risks. In addition, the proposed control measures were determined.
\end{abstract}


Keywords: Mechanical, Risks, Safety, Occupational, Health.

\section{Resumen}

El análisis de los riesgos laborales dentro de las empresas destinas a las obras civiles son de gran relevancia, más aún si se trata de garantizar la Seguridad y Salud Laboral de sus colaboradores. El presente trabajo investigativo se orienta al análisis de la situación actual por medio de la metodología de William Fine, donde se logra identificar y evaluar los riegos mecánicos, ya que la ocurrencia de éstos eventos catastróficos genera múltiples consecuencias, tanto para el empleador como para el empleado, los mismos que van desde el ausentismo laboral, lesiones físicas y mentales, hasta pérdidas económicas significativas para las empresas por los diversos casos de demandas o tratamientos que deben incurrir debido a la carencia de procedimientos de control y prevención, sean éstos de equipos y maquinarias dotados para los trabajos en la fase de fundición de estructuras metálicas, especialmente en la empresa Quintana Construcciones, los resultados obtenidos a través de la metodología cuali-cuantitativa aplicada permite tener las perspectivas de los trabajadores, quienes manifiestan estar condicionado a trabajos de alto riesgo mecánico, donde la falta de equipos de protección, así como la debida socialización de los lineamientos internos de seguridad laboral limita el involucramiento de los colaboradores, elemento esencial para mitigar la ocurrencia de los eventos de riesgos en la empresa en mención. Como parte de las conclusiones se determina la necesidad de diseñar una matriz de riesgos e identificar las medidas de control de riesgos existentes, así como de los propuestos.

Palabras Clave: Riesgos, Mecánicos, Seguridad, Salud, Laboral.

\section{Introduction}

De manera general, las condiciones de trabajo son un pilar fundamental dentro de las actividades laborales, tal es así, que la misma determina la calidad de vida del individuo, por lo que es imprescindible tener presente los factores que pueden generar riesgo o que se conviertan en una condición riesgosa con un evento catastrófico. Carbonel Slam \& Torres Valle (2011) en su artículo publicado en la Revista Scielo, con la temática "Evaluación de percepción de riesgo ocupacional" menciona que: "la inadecuada percepción de riesgo puede llegar a comprometer la ejecución de una actividad laboral", por consiguiente, las empresas u organizaciones debe tener previsto la aplicación de planes de intervención que ayuden a mitigar los riesgos laborales.

Al relacionar términos comunes dentro de la actividad laboral, en este caso los trabajadores, resulta indispensable reconocer la importancia que 
tiene la salud ocupacional. Ante aquello, según la Organización Mundial de la Salud, la salud ocupacional "es una disciplina orientada a dirigir y proteger la salud de los trabajadores mediante la adecuada prevención y control efectivo de enfermedades, accidentes, eliminación de los factores y condiciones riesgosas que ponen en peligro la salud y seguridad laboral".

Según la Organización Internacional del Trabajo- OTI (2014) aproximadamente "cada 15 segundos, un trabajador muere a causa de los accidentes o enfermedades que se relacionan con la actividad laboral, por ende cada 15 segundos, 153 trabajadores tienen un accidente laboral'. Para la OTI, la prevalencia de los accidentes laborales o de riego laboral pueden llegar a disminuirse, si y solo sí las empresas lleguen hacer conciencia de la magnitud y consecuencias de los accidentes, las múltiples lesiones y enfermedades relacionadas con el trabajo.

La importancia de la salud en el trabajo se llega a apreciar, si se considera la realidad de la sociedad, donde más de la mitad de la población se encuentra sujeta a cualquier actividad laboral, lo que implica su exposición directa a los riesgos de trabajo en industrias o empleos inadecuados. A nivel macro, las enfermedades laborales tienen incidencias desfavorables para la organización, la sociedad, así como para la familia del afectado; ya que dicho padecimiento se ve reflejado en su baja productividad, los gastos económicos generados por la atención médica, la hospitalización, las compensaciones salariales y el suministro de medicamentos, que en su gran mayoría pudieron ser evitados a través de los planes de intervenciones de riesgos laborales.

Sánchez Aguilar, Betzabé Pérez, y González Díaz (2011) aseguran que: "el trabajo requiere de leyes y reglamentos debidamente normados por un ente especializado, a la vez que debe ser cumplido por los empresarios y trabajadores", la salud laboral depende

del trabajo en equipo, de la puesta en marcha de programas institucionales, que previa coordinación determinen los factores y riesgos laborales al que son sometidos sus colaboradores y establezcan las medidas óptimas para hacer frente a esta situación.

Según las cifras expuestas por el Instituto Mexicano de Seguro Social (IMSS), (s.f.) "el elevado porcentaje de hospitalizaciones y tratamientos ambulatorios primarios están relacionados a las exposiciones de los trabajadores a los diferentes riesgos laborales". Sin lugar a dudas, el empleo se constituye en uno de los elementos fundamentales para el bienestar de las personas, así como un indicador determinante en la salud del trabajador, por lo tanto, es imprescindible considerar que los diversos lugares donde se genera trabajo se constituyen en una fuente inevitables de riesgos debido a la escasa participación del colectivo laboral. Dentro de las determinantes de los factores de riesgos laborales; los riesgos mecánicos representados por 
objetos, máquinas, equipos y herramientas llegan a ocasionar accidentes laborales, sea ésta por la falta de mantenimiento preventivo y/o correctivo, así como la carencia de normas de seguridad en el sistema de transmisión de fuerza, punto de operación, partes móviles, salientes o la falta de elementos de protección personal, situación que conlleva a niveles extremadamente riesgosos para la salud ocupacional (Organización Internacional de Trabajo, 2013).

Para la Universidad Carlos III de Madrid (2014) los riesgos mecánicos "son el conjunto de factores físicos que pueden estar asociados al inicio de una lesión por la acción mecánica de ciertas máquinas, herramientas, piezas a trabajar o materiales de proyección, así como sólidos o fluidos".

Crisanto \& Echeverría (2015) citan el Boletín estadístico No. 18 del año 2010, emitido por el Instituto Ecuatoriano de Seguridad Social (IESS) donde se registró "7.905 accidentes laborales, de los cuales 273 tuvo secuelas fatales en los afiliados". Las proyecciones presentadas en dicho informe refleja la realidad del sistema laboral en el país, donde la carencia de planes de intervención sobre riesgos mecánicos incrementa el riesgo de sufrir algún tipo de afectación a la salud.

Ante aquello, el presente estudio se enfoca en el análisis de las actividades u obras civiles que realiza la empresa Quintana construcciones, donde los requerimientos del uso de maquinarias e implementos para realizar fundiciones de estructuras de hormigón condiciona el bienestar de sus colaboradores, tal es así que ellos están expuestos a riesgos de índole mecánicos que ponen en juego la salud ocupacional de la empresa.

Se determina el análisis específico de los riesgos mecánicos al que están expuestos los trabajadores de dicha empresa, ya sean éstos por cizallamiento, atrapamiento o de arrastre, de aplastamiento, de sólidos y de líquidos, adicionando los peligros de corte, de seccionamiento, entre otros.

El problema de estudio surge por la existencia de diversas maquinarias que se encuentran en sus últimos años de vida útil, donde el cambio es necesario, lo que implica la exposición de los trabajadores a sufrir accidentes relacionados al mal funcionamiento de aquellos implementos. Así como el incremento de las lesiones o daños a la integridad física de los mismos relacionados a la inoperatividad de las herramientas o la carencia de procedimientos para su correcto uso.

\section{Objetivo General}

Evaluar los riesgos mecánicos a través de la metodología de Willian Fine para contribuir a mejorar la Seguridad y Salud Laboral de los trabajadores que realizan fundición de estructuras metálicas en la empresa Quintana Construcciones. 


\section{Objetivos Específicos}

- Identificar cómo la carencia de procedimientos para controles preventivos incide en el índice de riesgos mecánicos al que se encuentran expuestos los trabajadores de la fase de fundición de estructuras metálicas de la empresa Quintana Construcciones. Evaluar los riesgos mecánicos que provocan lesiones y daños a la integridad física de los trabajadores de la fase de fundición de estructuras metálicas de la empresa Quintana Construcciones.

- Diseñar un programa de intervención que reduzcan los riesgos mecánicos en la fase de fundición de estructuras de metálicas que realiza la empresa Quintana Construcciones.

\section{Metodología}

El presente estudio se desarrolla dentro del diseño cuali-cuantitativo, donde se estableció el análisis de la percepción de los factores de riesgos por parte de los colaboradores, así como la identificación de los factores de riesgos inherentes e intrínsecos en toda la actividad, por lo que surge la incertidumbre de los posibles eventos que condicionan el trabajo.

Ante aquello, la determinación de los peligros se dio por medio de la identificación de las actividades que realizan las personas que trabajan en la Empresa Quintana Construcciones, es decir que cada uno de los procesos conlleva a la determinación de múltiples peligros para los trabajadores, lo que requirió de una secuencia lógica de actividades para determinar de manera clara y objetiva la peligrosidad de un proceso.

Por lo tanto, el análisis situacional permitirá generar un plan de intervención cuyas medidas de protección a tomar servirán para mitigar las probabilidades de sufrir accidentes por riesgos mecánicos y optimizar la productividad de la empresa, manteniendo una excelente salud ocupacional.

En cuanto a los resultados obtenidos con la aplicación de la encuesta a los trabajadores de la fase de fundición de la Empresa Quintana Construcciones, se logra determinar la necesidad de aplicar una identificación y evaluación de los riegos mecánicos a través de la metodología de William Fine, considerada éste como la base para el plan de intervención que mitigue los índices de ocurrencia de accidentes mecánicos.

\section{Hipótesis}

El análisis y evaluación de los riesgos mecánicos a través del método de Willian Fine permite mejorar la Seguridad y Salud Laboral en los trabajadores de la empresa Quintana Construcciones que realizan fundición de estructuras metálicas. 


\section{Universo}

El universo de estudio se encuentra conformado por todos los trabajadores que mantienen una actividad directa con equipos $\mathrm{y}$ herramientas, sean estas manuales o mecánicas, los mismos que están expuestos a una diversidad de riesgos mecánicos que atentan en contra de la salud ocupacional dentro de la organización.

\section{Muestra}

La muestra es establecida por un total de 40 trabajadores, a lo que se considera como una población finita, determinado como carácter no probabilístico dado el caso y necesidad de participación.

Los resultados obtenidos por medio de la encuesta y otros instrumentos aplicados fueron tabulados y procesados a través de una herramienta informática denominada Excel. Los resultados se presentan mediante la representación de figuras con su respectivo porcentajepara posteriormente ser mostrados por medio de tablas y gráficos estadísticos que facilitan su análisis e interpretación, así como la verificación de las hipótesis. Dentro de este proceso se realizaron 2 sesiones con todos los colaboradores, donde primero se informó y explico al personal sobre el formulario de preguntas (Anexo 1 formulario de preguntas).

El mismo está elaborado de manera que sea entendió por todas las personas con respuestas de si, no, (n/s) no sabe o (n/p) no procede; se tiene como referencia que la mayor parte del personal tiene como nivel educativo estudios primarios. La segunda sección se extendió el formulario a los presente para que sean contestados.

La gestión de datos fue realizada a través de las siguientes acciones:

- La descripción de los datos a tabular a través de la construcción de tablas.

- La distribución gráfica de los mismos mediante pasteles estadísticos donde se plasma sus respectivos porcentajes.

Por lo tanto, a través de la estadística descriptiva, así como el desarrollo de la matriz de identificación y evaluación de riesgos se logró determinar la valoración de los riesgos mecánicos en la empresa Quintana Construcciones.

\section{Resultados}

A través de los instrumentos de investigación se logró determinar una serie de indicadores que pone en manifiesto la exposición de riesgos mecánicos a los trabajadores de la fase de fundición de estructuras metálicas en la Empresa Quintana Construcciones, entre las que se encuentran:

Análisis De La Encuesta Realizada A Los Trabajadores De La Fase De Fundición De Estructuras Metálicas De La Empresa Quintana Construcciones 
1.- Altura de la superficie de trabajo inadecuada para el tipo de tarea o para las dimensiones del trabajador

\begin{tabular}{|c|c|c|}
\multicolumn{4}{|c}{ Tabla 1. Distribución sumatoria } \\
\hline OPCIONES & RESULTADOS & PORCENTAJES \\
\hline SI & 21 & $52 \%$ \\
\hline NO & 14 & $35 \%$ \\
\hline NO SABE & 5 & $13 \%$ \\
\hline NO PROCEDE & 0 & $0 \%$ \\
\hline TOTAL & $\mathbf{4 0}$ & $\mathbf{1 0 0 \%}$ \\
\hline
\end{tabular}

Elaborado por: Equipo de trabajo

Fuente: Investigación de campo

Altura de la superficie de trabajo inadecuada para el tipo de tarea 0 para las dimensiones del trabajador

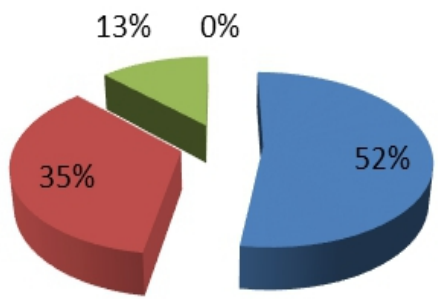

Grafico 1. Distribución porcentual

Elaborado por: Equipo de trabajo

Fuente: Investigación de campo

Análisis.- Los resultados obtenidos demuestran que el $52 \%$ de los trabajadores consideran que la altura de la superficie de trabajo es inadecuada para este tipo de tarea, así como su dimensionamiento, lo que pone en riesgo la integridad física del personal encargado de este tipo de actividad, mientras que el $35 \%$ responde que no y el $13 \%$ que no sabe.

\section{2.- Espacio de trabajo insuficiente o inadecuado}

Tabla 2. Distribución sumatoria

\begin{tabular}{|c|c|c|}
\hline OPCIONES & RESULTADOS & PORCENTAJES \\
\hline SI & 18 & $45 \%$ \\
\hline NO & 22 & $55 \%$ \\
\hline NO SABE & 0 & $0 \%$ \\
\hline NO PROCEDE & 0 & $0 \%$ \\
\hline TOTAL & $\mathbf{4 0}$ & $\mathbf{1 0 0 \%}$ \\
\hline
\end{tabular}

Elaborado por: Equipo de trabajo

Fuente: Investigación de campo 


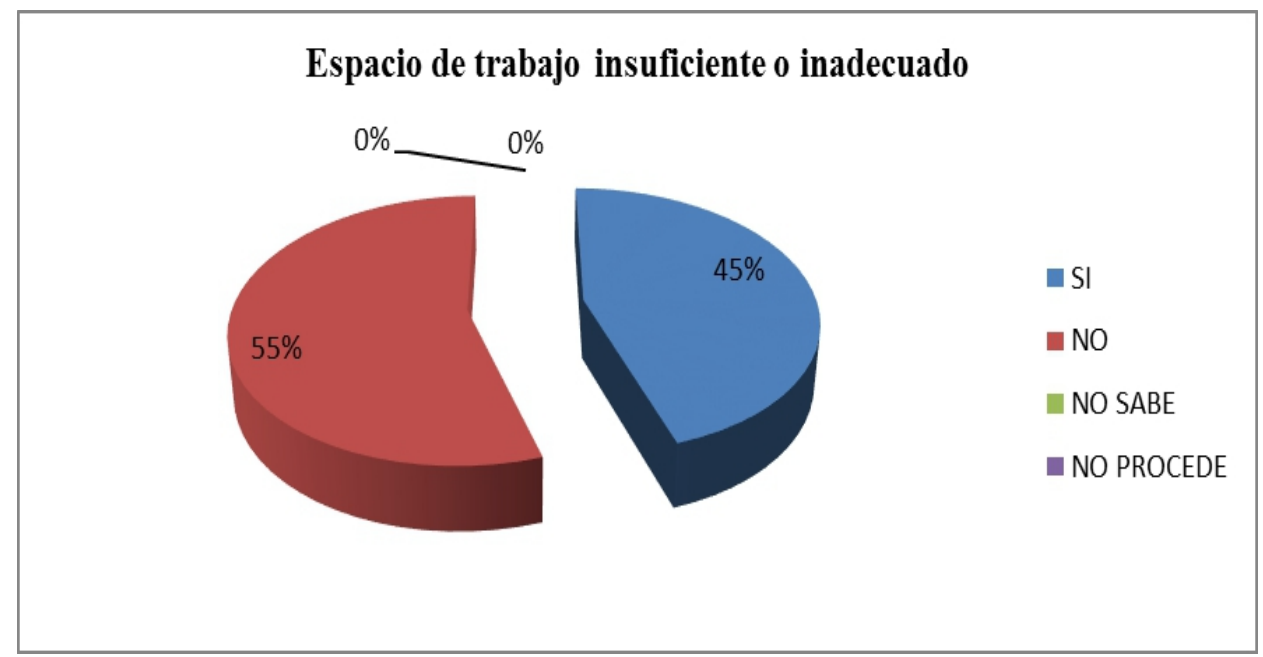

Gráfico 2. Distribución porcentual

Elaborado por: Equipo de trabajo

Fuente: Investigación de campo

Análisis.- Los resultados obtenidos demuestran que el $45 \%$ de los trabajadores consideran que el espacio de trabajo es insuficiente e inadecuado, por lo que su actividad la realizan en condiciones mínimas para un desempeño laboral más eficiente, mientras que el 55\% responde que no. A pesar de aquello, existen una percepción negativa en relación al área de trabajo, que sumado a los riesgos mecánicos deterioran la Seguridad y Salud laboral de los colaboradores.

3.- Los controles y los indicadores asociados a su trabajo (mandos de los equipos, tableros de instrumentación, etc.) se visualizan con dificultad

Tabla 3. Distribución sumatoria

\begin{tabular}{|c|c|c|}
\hline OPCIONES & RESULTADOS & PORCENTAJES \\
\hline SI & 10 & $33 \%$ \\
\hline NO & 15 & $50 \%$ \\
\hline NO SABE & 5 & $17 \%$ \\
\hline NO PROCEDE & 0 & $0 \%$ \\
\hline TOTAL & $\mathbf{4 0}$ & $\mathbf{1 0 0 \%}$ \\
\hline
\end{tabular}

Elaborado por: Equipo de trabajo

Fuente: Investigación de campo 


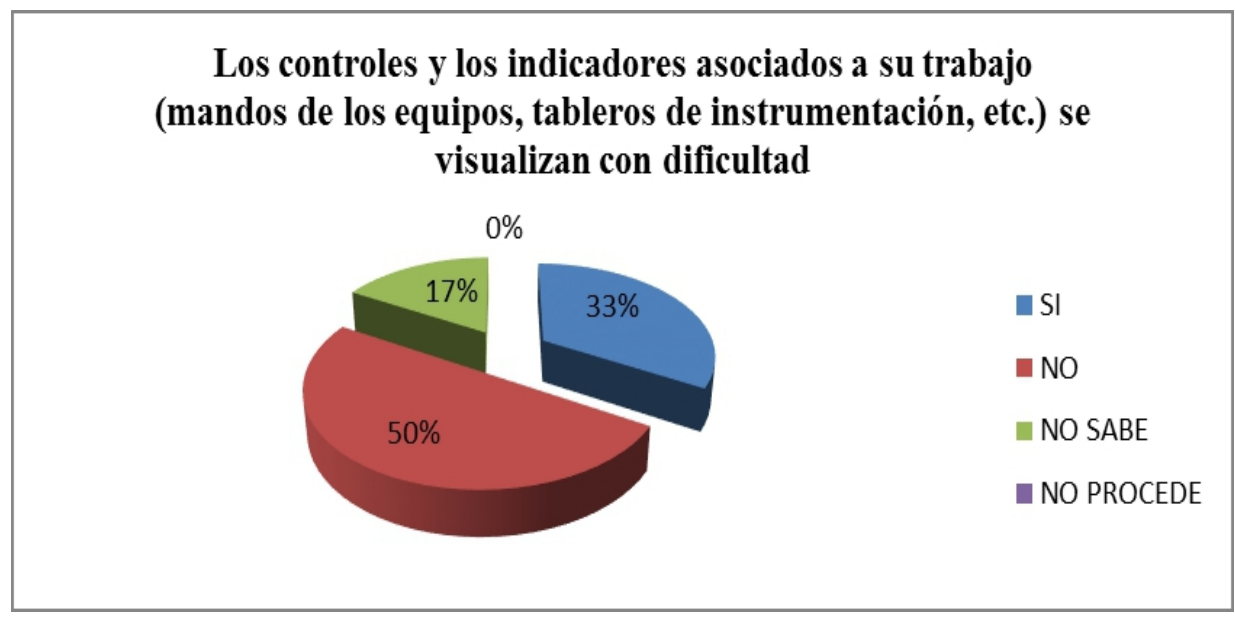

\section{Gráfico 3. Distribución porcentual \\ Elaborado por: Equipo de trabajo \\ Fuente: Investigación de campo}

Análisis.- Los resultados obtenidos demuestran que el $33 \%$ de los trabajadores expresan que los controles y los indicadores asociados a su trabajo se visualizan con dificultad, es decir que el personal de la fase de fundición de estructuras metálicas se encuentran limitados en conocer ciertas normas preventivas dentro del área de trabajo aumentando la posibilidad de sufrir accidentes relacionados a los riesgos mecánicos, mientras que para el $50 \%$ no existe este condicionamiento y el $17 \%$ no lo sabe.

\section{4.- Zonas de trabajo y lugares de paso dificultados por exceso de objetos}

Tabla 4. Distribución sumatoria

\begin{tabular}{|c|c|c|}
\hline OPCIONES & RESULTADOS & PORCENTAJES \\
\hline SI & 29 & $72 \%$ \\
\hline NO & 11 & $28 \%$ \\
\hline NO SABE & 0 & $0 \%$ \\
\hline NO PROCEDE & 0 & $0 \%$ \\
\hline TOTAL & $\mathbf{4 0}$ & $\mathbf{1 0 0 \%}$ \\
\hline
\end{tabular}

Elaborado por: Equipo de trabajo

Fuente: Investigación de campo 


\section{Zonas de trabajo y lugares de paso dificultados por exceso de} objetos

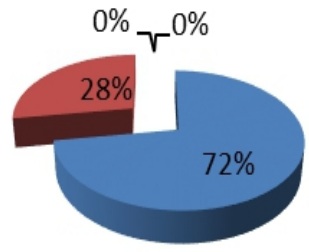

$\square \mathrm{SI}$

NO

NO SABE

NO PROCEDE

\section{Gráfico 4. Distribución porcentual \\ Elaborado por: Equipo de trabajo \\ Fuente: Investigación de campo}

Análisis.- Los resultados obtenidos demuestran que el $72 \%$ de los trabajadores expresan que si existe un dificultad al paso en las diversas zonas y puestos de trabajo por el exceso de objetos, factor elemental para establecer la carencia de involucramiento de los colaboradores para cumplir con las normas de seguridad y salud laboral aumentando las posibilidades de sufrir accidentes de índole mecánica, mientras que el 28\% expresa que no.

5.- Ruidos ambientales molestos o que provocan dificultad en la concentración para la realización del trabajo

Tabla 5. Distribución sumatoria

\begin{tabular}{|c|c|c|}
\hline OPCIONES & RESULTADOS & PORCENTAJES \\
\hline SI & 31 & $77 \%$ \\
\hline NO & 9 & $23 \%$ \\
\hline NO SABE & 0 & $0 \%$ \\
\hline NO PROCEDE & 0 & $0 \%$ \\
\hline TOTAL & $\mathbf{4 0}$ & $\mathbf{1 0 0 \%}$ \\
\hline
\end{tabular}

Elaborado por: Equipo de trabajo

Fuente: Investigación de campo

Ruidos ambientales molestos o que provocan dificultad en la concentración para la realización del trabajo

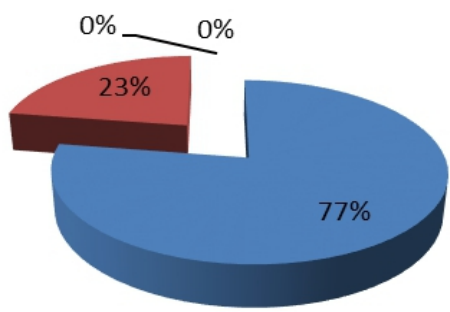


Análisis.- Los resultados obtenidos demuestran que el $77 \%$ de los trabajadores expresan que si existe un alto índice de ruido ambiental que provoca dificultad en la concentración del trabajo, los mismos que son consecuente a las características de la obra civil que se realiza, lo que amerita el uso del equipo necesario para mitigar los excesivos niveles de contaminación acústicas presentes en los puestos de trabajo y que mucho de ellos son inherentes a la actividad realizada, mientras que el $9 \%$ manifiesta que no.

\section{6.- Existen reflejos o deslumbramientos molestos en el puesto de trabajo o su entorno}

Tabla 6. Distribución sumatoria

\begin{tabular}{|c|c|c|}
\hline OPCIONES & RESULTADOS & PORCENTAJES \\
\hline SI & 27 & $67 \%$ \\
\hline NO & 13 & $33 \%$ \\
\hline NO SABE & 0 & $0 \%$ \\
\hline NO PROCEDE & 0 & $0 \%$ \\
\hline TOTAL & $\mathbf{4 0}$ & $\mathbf{1 0 0 \%}$ \\
\hline
\end{tabular}

Elaborado por: Equipo de trabajo

Fuente: Investigación de campo

\section{Existen reflejos o deslumbramientos molestos en el puesto de trabajo o su entorno}

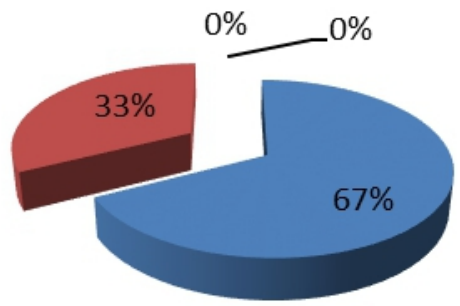

$\square \mathrm{SI}$

NO

NO SABE

- NO PROCEDE

\section{Gráfico 6. Distribución porcentual \\ Elaborado por: Equipo de trabajo \\ Fuente: Investigación de campo}

Análisis.- Los resultados obtenidos demuestran que el $67 \%$ de los trabajadores expresan que si existe exposiciones de deslumbramientos y reflejos en los puestos de trabajos, los mismos que pone en riesgo la operatividad de equipos y maquinarias ante la reacción humana a dicho evento, factor elemental que incide en los índices de ocurrencia de riesgos mecánicos, mientras que para el 33\% esta causalidad no existe. Es decir que se debe proporcionar los equipos necesarios para evitar dichas exposiciones, como lo son gafas de protección. 
7.- Se manejan equipos de trabajo o herramientas peligrosas, defectuosas o en mal estado

Tabla 7. Distribución sumatoria

\begin{tabular}{|c|c|c|}
\hline OPCIONES & RESULTADOS & PORCENTAJES \\
\hline SI & 22 & $55 \%$ \\
\hline NO & 18 & $45 \%$ \\
\hline NO SABE & 0 & $0 \%$ \\
\hline NO PROCEDE & 0 & $0 \%$ \\
\hline TOTAL & $\mathbf{4 0}$ & $\mathbf{1 0 0 \%}$ \\
\hline
\end{tabular}

Elaborado por: Equipo de trabajo

Fuente: Investigación de campo

Se manejan equipos de trabajo o herramientas peligrosas, defectuosas 0 en mal estado

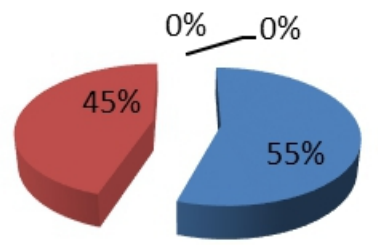

SI

NO

NO SABE

- NO PROCEDE

\section{Gráfico 7. Distribución porcentual \\ Elaborado por: Equipo de trabajo \\ Fuente: Investigación de campo}

Análisis.- Los resultados obtenidos demuestran que el $55 \%$ de los trabajadores expresan que si se manejan equipos defectuosos o herramientas peligrosas, por lo que se debe tomar en consideración los procesos de controles preventivos y correctivos a la maquinaria que utiliza el personal de la fase de fundición con el objetivo de mitigar la exposición a riesgos mecánicos, mientras que el 455 expresa que no.

8.- Carece de instrucciones de trabajo, en lenguaje comprensible para los trabajadores en relación al uso de los equipos o herramientas

Tabla 8. Distribución sumatoria

\begin{tabular}{|c|c|c|}
\hline OPCIONES & RESULTADOS & PORCENTAJES \\
\hline SI & 14 & $35 \%$ \\
\hline NO & 26 & $65 \%$ \\
\hline NO SABE & 0 & $0 \%$ \\
\hline NO PROCEDE & 0 & $0 \%$ \\
\hline TOTAL & $\mathbf{4 0}$ & $\mathbf{1 0 0 \%}$ \\
\hline
\end{tabular}

Elaborado por: Equipo de trabajo

Fuente: Investigación de campo 


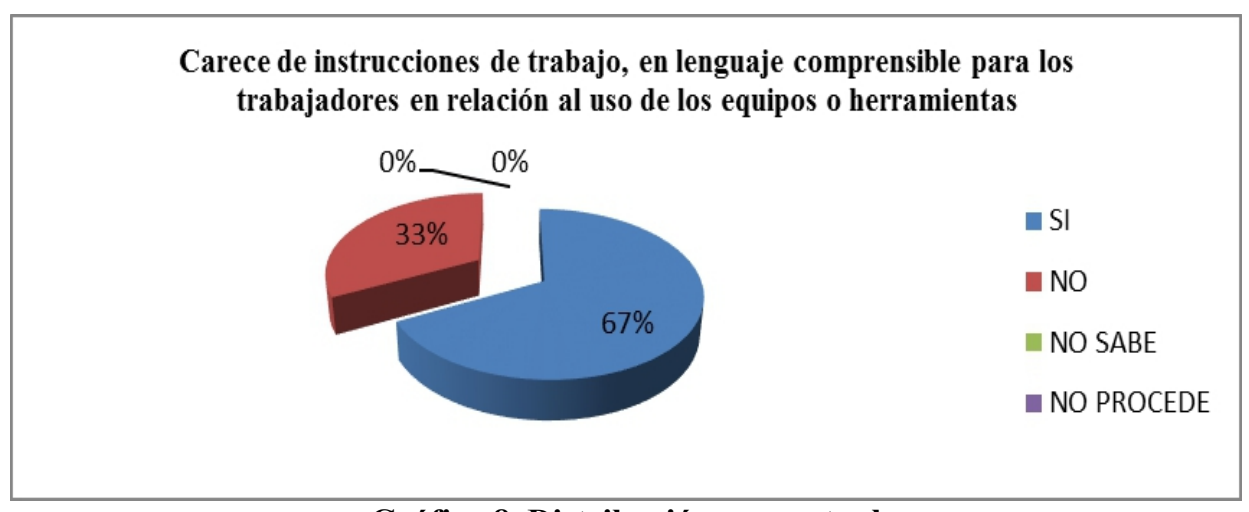

\section{Gráfico 8. Distribución porcentual \\ Elaborado por: Equipo de trabajo \\ Fuente: Investigación de campo}

Análisis.- Los resultados obtenidos demuestran que el $35 \%$ de los trabajadores expresan que si carece de instrucciones de trabajo, lenguaje comprensible para el uso de equipos o herramientas dentro de su actividad laboral, mientras que el $65 \%$ expresa que no. A pesar de aquello, la primera cifra debe ser considerada como un punto de inflexión para la toma de decisiones relacionadas al mejoramiento de la Seguridad y Salud Laboral en la fase de fundición de estructuras metálicas en la empresa en cuestión.

\section{9.- Carencia de procedimientos de control en el funcionamiento de equipos y maquinarias}

Tabla 9. Distribución sumatoria

\begin{tabular}{|c|c|c|}
\hline OPCIONES & RESULTADOS & PORCENTAJES \\
\hline SI & 27 & $67 \%$ \\
\hline NO & 13 & $33 \%$ \\
\hline NO SABE & 0 & $0 \%$ \\
\hline NO PROCEDE & 0 & $0 \%$ \\
\hline TOTAL & $\mathbf{4 0}$ & $\mathbf{1 0 0 \%}$ \\
\hline
\end{tabular}

Elaborado por: Equipo de trabajo

Fuente: Investigación de campo

\section{Carencia de procedimientos de control en el funcionamiento de equipos y maquinarias}

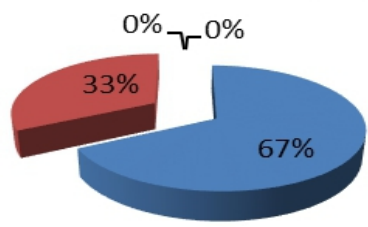

$$
\begin{aligned}
& =\text { SI } \\
& =\text { NO } \\
& =\text { NO SABE } \\
& =\text { NO PROCEDE }
\end{aligned}
$$

Gráfico 9. Distribución porcentual

Elaborado por: Equipo de trabajo

Fuente: Investigación de campo 
Análisis.- Los resultados obtenidos demuestran que el $67 \%$ de los trabajadores expresan que si existe una carencia de procedimientos de control en el funcionamiento de los equipos y maquinarias, lo que demuestra la exposición relativa a los diversos riesgos mecánicos debido al fallo en la instrumentación, mientras que el $33 \%$ expresa que no, criterio considerado como alejado de la realidad y que debe ser evaluado a través del método Fine.

\section{Contrastación Empírica}

Entre los indicadores evaluados se encuentra como punto relevante la carencia de procedimientos de controles preventivos y correctivos de herramientas y equipos, donde la probabilidad de ocurrencia de eventos catastróficos para la Seguridad y Salud laboral es significativa. Esto contrasta con el estudio realizado por la Fundación para la Prevención de Riesgos Laborales en el Sector Metal en obras de Construcción (2011), donde establece que "los niveles de incidencia mortales en los trabajadores del área de construcción civil, así como de peones en general se deben al mal manejo y operatividad de los equipos y herramientas de trabajo". (Cepyme Aragón, 2011)

Bajo este contexto, los índices de siniestralidad ocasionados por los riesgos mecánicos son generalizados en casi todas las empresas dedicadas a la obra civil, donde la carencia de procedimientos de control en los equipos y herramientas conlleva a una exposición eminente del personal colaborativo a sufrir accidentes laborales que atenten con su integridad física y mental.

Por otro lado, el estudio efectuado por el Instituto Nacional de Seguridad e Higiene en el Trabajo- INSHT (2011) reporta un total de 32.319 accidentes ocurridos en horas de trabajo, los mismos que corresponden al sector de construcción y metal (Cepyme, 2011). Ante aquello, se ponen en consideración la ocurrencia progresiva de accidentes laborales en las áreas de construcción y fundición, lo que implica la intervención oportuna para mitigar la ocurrencia de riesgos mecánicos.

La serie de exposiciones a los riesgos mecánicos incide en los niveles de accidentabilidad en las diversas empresas dedicadas a la obra civil, lo que implica la inspección progresiva de las áreas, equipos y herramientas antes de las fases de operatividad, es decir que la necesidad de poseer procedimientos sobre el manejo adecuado de los mismos responden a los criterios de Seguridad y Salud Laboral.

De forma adicional, el uso adecuado de equipos de protección personal son producto de la identificación y evaluación de los riesgos mecánicos en el área de trabajo (Homero de Fundiciones, 2013).

El estudio realizado por (Rodríguez Garzón, Martínez Fiestas, \& López Alonso, 2012) como parte del artículo publicado en la Revista de la 
Construcción con la temática El riesgo percibido por el trabajador de la construcción: ¿qué rol juega el oficio? Manifiesta que: "es preciso tomar en consideración los aspectos conductuales cuando el individuo decide trabajar en ambientes inseguros"; como parte del estudio se muestra que el 83,9\% de los riesgos laborales son percibidos como parte de la experiencia de trabajo, la formación adquirida y los conocimientos previos.

Es decir que el análisis obtenido a través de la encuesta aplicada a puntos específicos deben ser considerados como relevantes para el planteamiento de un plan de intervención que ayude a mitigar los efectos negativos de los riesgos mecánicos percibidos por el personal colaborativo como parte compensatoria a la Seguridad y Salud Laboral.

Entre las diferencias identificadas por los referentes empíricos, el presente estudio toma en consideración para el planteamiento del plan de intervención la percepción del personal colaborativo, quienes en base a su experiencia y estados conductuales expresan la frecuencia de riesgos al que son sometidos, ayudando de esta forma a la toma de decisiones que logre los correctivos necesarios.

Cuadro 1. Identificación, estimación cualitativa y control de riesgos-método de William Fine

\begin{tabular}{|c|c|c|c|c|c|c|c|c|c|c|c|c|c|c|}
\hline $\begin{array}{l}\text { AREA } \\
\text { UBICACION }\end{array}$ & PROCESO & ACTIVIDAD & $\begin{array}{c}\text { TIPODE } \\
\text { ACTIVIDAD }\end{array}$ & $\begin{array}{l}\text { FACTORES DE } \\
\text { RIESGO }\end{array}$ & TIPO DERIESGO & $\begin{array}{l}\text { EEECTOS POSIBLES, REALESY } \\
\text { POTENCIALES }\end{array}$ & CARGOS EXPUESTOS & 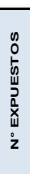 & 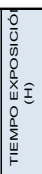 & 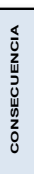 & 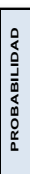 & $\begin{array}{l}z \\
z \\
\frac{0}{0} \\
\frac{0}{2} \\
\frac{0}{x} \\
\text { un }\end{array}$ & i & $\begin{array}{c}\mid \text { INTERPRETACIÓN } \\
\text { GP (INT 1) }\end{array}$ \\
\hline & \multirow{4}{*}{$\begin{array}{l}\text { MOVIMIENTO DE } \\
\text { TIERRA }\end{array}$} & Preparación de excavadora & RUTINARIO & MECANICO & $\begin{array}{c}\text { Caida de distinto nivel al subir en } \\
\text { la máquina }\end{array}$ & $\begin{array}{l}\text { Lesiones, Golpes y fracturas } \\
\text { mayores }\end{array}$ & OPERADOR & 0 & 8 & 6 & 7 & 10 & 420 & MEDAA \\
\hline & & Circulación de volquetas & RUTINARIO & MECANICO & Arrollamiento de personas & Golpes, tracturas y muerte & OBREROS & 10 & 8 & 10 & 7 & 10 & 700 & ALTA \\
\hline & & $\begin{array}{l}\text { Traslado de excavadora a sitio de } \\
\text { trabajo }\end{array}$ & RUTINARIO & MECANICO & Topografia irregular & $\begin{array}{c}\text { Daños materiales y lesiones, golpes } \\
\text { y fracturas }\end{array}$ & OPERADOR & 10 & 8 & 4 & 4 & 2 & 32 & BAJA \\
\hline & & $\begin{array}{l}\text { Excavación de zanja } 70 \mathrm{~m} 3 \text { con } \\
\text { retroexcavadora }\end{array}$ & RUTINARIO & MECANICO & Arrollamiento de personas & Golpes, fracturas y muerte & OBREROS & 10 & 8 & 10 & 7 & 10 & 700 & ALTA \\
\hline & \multirow{4}{*}{ TOPOGRAFIA } & $\begin{array}{l}\text { Ubicación de equipo topografico } \\
\text { (Estación Total o Nivel) }\end{array}$ & RUTINARIO & MECANICO & $\begin{array}{l}\text { Golpe con objeto por } \\
\text { despredimiento }\end{array}$ & $\begin{array}{l}\text { Lesiones, Golpes y fracturas } \\
\text { mayores }\end{array}$ & TOPOGRAFO & 4 & 8 & 1 & 4 & 10 & 40 & BAJA \\
\hline & & $\begin{array}{l}\text { Levantamiento de información } \\
\text { georeferenciado y toma de niveles }\end{array}$ & RUTINARIO & MECANICO & $\begin{array}{l}\text { Caida a distinto nivel y mismo } \\
\text { nivel por topografia irregular }\end{array}$ & $\begin{array}{l}\text { Lesiones, Golpes y fracturas } \\
\text { mayores }\end{array}$ & TOPOGRAFO & 4 & 8 & 4 & 7 & 10 & 280 & BAJA \\
\hline & & $\begin{array}{l}\text { Traslado de equipo para toma de } \\
\text { lectura (prisma o nivel) }\end{array}$ & RUTINARIO & MECANICO & $\begin{array}{l}\text { Caida a distinto nivel y mismo } \\
\text { nivel por topografia irregular }\end{array}$ & $\begin{array}{l}\text { Lesiones, Golpes y fracturas } \\
\text { mayores }\end{array}$ & CADENERO & 4 & 8 & 6 & 7 & 10 & 420 & MEDAA \\
\hline & & $\begin{array}{l}\text { Posecionamiento con prisma o } \\
\text { bastón en sitio para toma de lectura }\end{array}$ & RUTINARIO & MECANICO & $\begin{array}{l}\text { Golpe con objeto por } \\
\text { despredimiento }\end{array}$ & Lesiones y Golpes & CADENERO & 4 & 8 & 1 & 4 & 10 & 40 & BAJA \\
\hline
\end{tabular}

Fuente: Investigación de campo

Elaborado por: Ing. Ind. Mendoza Proaño Jairón Enrique 


\section{Cuadro 2. Identificación y evaluación cualitativa y control de riesgos-método de}

\begin{tabular}{|c|c|c|c|c|c|c|c|c|}
\hline \multicolumn{4}{|r|}{ EVALUACIÓN } & \multicolumn{3}{|c|}{ CONTROLES } & \multicolumn{2}{|c|}{ MEDIDAS } \\
\hline 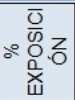 & 은 & $\underset{c}{\alpha}$ & $\begin{array}{l}\text { INTERPRETACION } \\
\text { GP (INT 2) }\end{array}$ & $\mathrm{F}$ & M & 1 & CONTROL DE RIESGOS EXISTENTE & CONTROL DE RIESGOS PROPUESTA \\
\hline $25 \%$ & 2 & 840 & BAJA & & & $x$ & $\begin{array}{l}\text { Uso de botas de cuero antideslizante. } \\
\text { Inducción sobre los riesgos Mecánicos y caídas a } \\
\text { distinto nivel. }\end{array}$ & $\begin{array}{l}\text { Elaboración de procedimiento para operación de } \\
\text { excavadora }\end{array}$ \\
\hline $25 \%$ & 2 & 1400 & BAJA & & $x$ & & $\begin{array}{l}\text { Colocación de señalización y confinación de áreas } \\
\text { existentes. } \\
\text { Inspección de maquinarias }\end{array}$ & $\begin{array}{l}\text { Elaboración procedimiento para manejo a la defensiva } \\
\text { (volquetas y vehículos). }\end{array}$ \\
\hline $25 \%$ & 2 & 64 & BAJA & & & $\mathrm{x}$ & $\begin{array}{l}\text { Capacitación a colaboradores sobre los riesgos en } \\
\text { charla pre-jornal }\end{array}$ & Generación de orden para movimiento de maquinaria \\
\hline $25 \%$ & 2 & 1400 & BAJA & $\mathrm{x}$ & & $\mathrm{x}$ & $\begin{array}{l}\text { Capacitación a colaboradores y operadores sobre } \\
\text { los riesgos en charla pre-jornal. } \\
\text { Inspección de maquinarias }\end{array}$ & $\begin{array}{l}\text { Aplicación prueba de alchochimetro a Operadores y } \\
\text { choferes. } \\
\text { Generación de procedimiento y registro para prueba } \\
\text { de alcochimetro. }\end{array}$ \\
\hline $10 \%$ & 1 & 40 & BAJA & & & $x$ & $\begin{array}{l}\text { Capacitación a colaboradores sobre los riesgos por } \\
\text { golpes con equipos y/o herramientas en charla pre- } \\
\text { jornal y Dominical }\end{array}$ & Anclaje de estación total \\
\hline $10 \%$ & 1 & 280 & BAJA & & & $\mathrm{x}$ & $\begin{array}{l}\text { Capacitación a colaboradores sobre los riesgos de } \\
\text { caída a distinto y mismo nivel en charla pre-jornal y } \\
\text { Dominical }\end{array}$ & \\
\hline $10 \%$ & 1 & 420 & BAJA & & & $x$ & $\begin{array}{l}\text { Capacitación a colaboradores sobre los riesgos de } \\
\text { caída a distinto y mismo nivel en charla pre-jornal y } \\
\text { Dominical }\end{array}$ & \\
\hline $10 \%$ & 1 & 40 & BAJA & & & $x$ & $\begin{array}{l}\text { Capacitación a colaboradores sobre los riesgos por } \\
\text { golpes con equipos y/o herramientas en charla pre- } \\
\text { jornal y Dominical }\end{array}$ & Anclaje de estación total \\
\hline
\end{tabular}

\section{William Fine (1)}

Fuente: Investigación de campo

Elaborado por: Ing. Ind. Mendoza Proaño Jairón Enrique 
Cuadro 3. Identificación y evaluación cualitativa y control de riesgos-método de William Fine

\begin{tabular}{|c|c|c|c|c|c|c|c|c|c|c|}
\hline 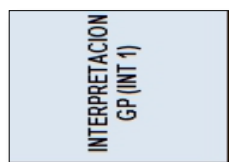 & 亮 & 蒿 & 喜 & 喜 & 喜 & 效 & 喜 & 善 & 须 & 喜 \\
\hline d's & צิ & ษิ & ్ำ & สูป & $\dddot{\circ}$ & ్ำ & สี & צิ & F & q \\
\hline NOIOISOdXヨ & 운 & $\infty$ & 0 & $\infty$ & 0 & 0 & 0 & 은 & 0 & 으 \\
\hline aマa। רוя & $n$ & - & r & n & n & - & $n$ & n & n & $\sigma$ \\
\hline VIONANOISNOO & $\infty$ & 우 & $\infty$ & $\infty$ & $\theta$ & $\infty$ & $\infty$ & $\infty$ & - & - \\
\hline (H) NOISISOdXa & $\infty$ & $\infty$ & $\infty$ & $\infty$ & $\infty$ & $\infty$ & $\infty$ & $\infty$ & $\infty$ & $\infty$ \\
\hline SOLSBndxa oN & $\infty$ & o) & $\infty$ & os & $\infty$ & o) & $\infty$ & $\infty$ & $\infty$ & $\infty$ \\
\hline 总管 & 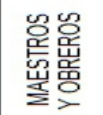 & 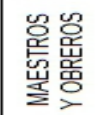 & 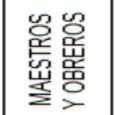 & 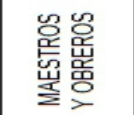 & 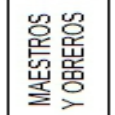 & 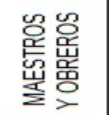 & 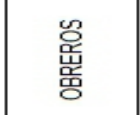 & 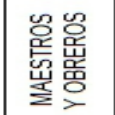 & 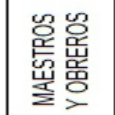 & $\begin{array}{l}\text { 足 } \\
\text { 点 } \\
\text { 尙 }\end{array}$ \\
\hline 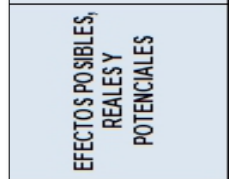 & 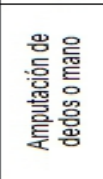 & 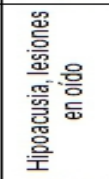 & 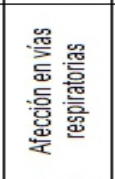 & 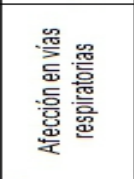 & $\begin{array}{l}\text { 흘 } \\
\text { 总 } \\
\text { 음 }\end{array}$ & 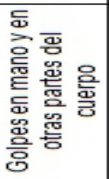 & 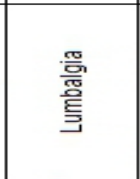 & 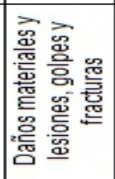 & 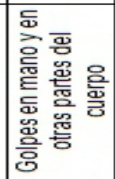 & 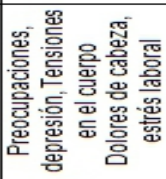 \\
\hline 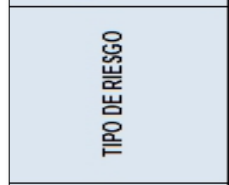 & 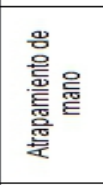 & 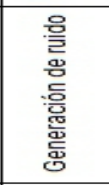 & 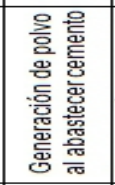 & 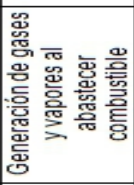 & 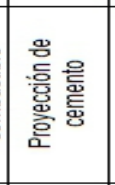 & 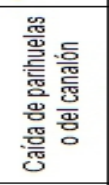 & 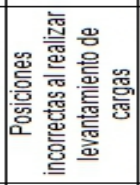 & 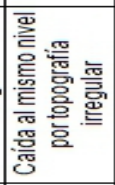 & 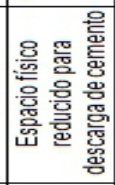 & 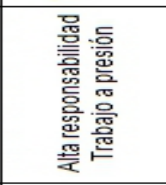 \\
\hline 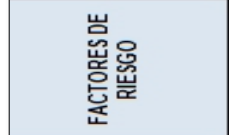 & 总 & $\frac{8}{\frac{0}{4}}$ & 高 & 高 & $\begin{array}{l}\text { 일 } \\
\text { 莣 } \\
\text { 至 }\end{array}$ & $\begin{array}{l}\text { 일 } \\
\text { 䓌 }\end{array}$ & $\begin{array}{l}\text { 읗 } \\
\text { 产 } \\
\text { 吕 }\end{array}$ & 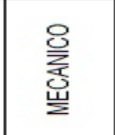 & $\begin{array}{l}\text { 禀 } \\
\text { 岸 }\end{array}$ & $\begin{array}{l}\frac{\overrightarrow{1}}{0} \\
\text { o } \\
\frac{0}{50}\end{array}$ \\
\hline 啱高 & $\begin{array}{l}\text { 울 } \\
\text { 景 } \\
\text { 胥 }\end{array}$ & 울 & $\begin{array}{l}\text { 울 } \\
\text { 景 } \\
\text { 䇂 }\end{array}$ & 울 & 을 & 을 & 을 & 울 & 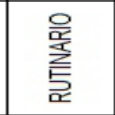 & 울 \\
\hline 릴 & 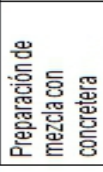 & 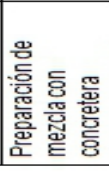 & 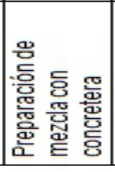 & 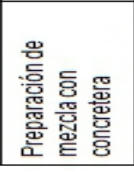 & 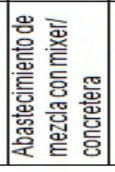 & 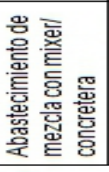 & 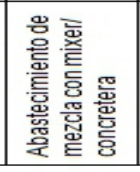 & 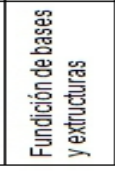 & 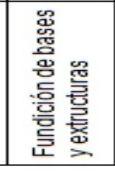 & 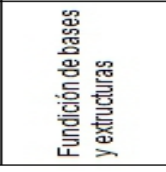 \\
\hline $\begin{array}{l}\text { 岕 } \\
\text { 适 }\end{array}$ & \multicolumn{10}{|c|}{ 흥 } \\
\hline
\end{tabular}

Elaborado por: Ing. Ind. Mendoza Proaño Jairón Enrique 
Cuadro 4. Identificación y evaluación cualitativa y control de riesgos-método de William Fine

\begin{tabular}{|c|c|c|c|c|c|c|c|c|c|c|c|}
\hline \multirow{2}{*}{ 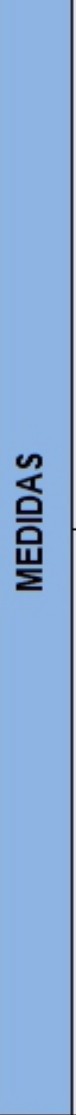 } & 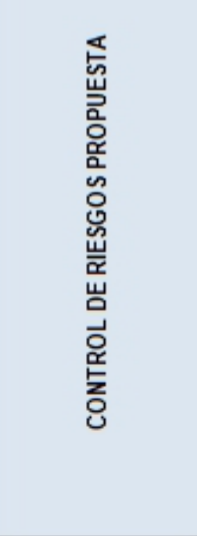 & & 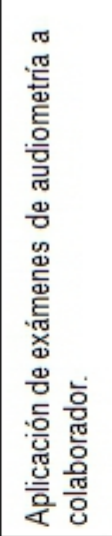 & & & & & & & & \\
\hline & 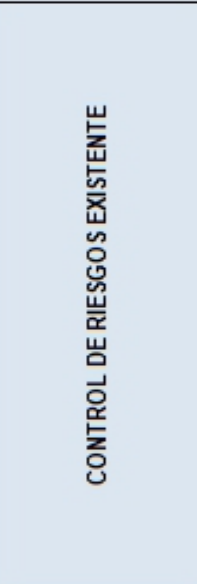 & 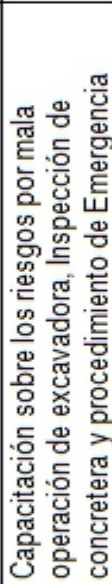 & 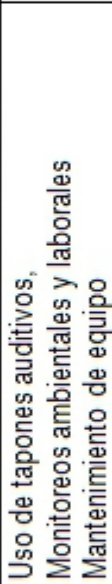 & 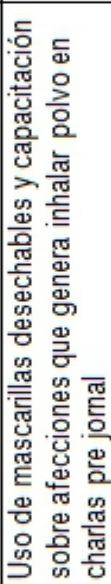 & 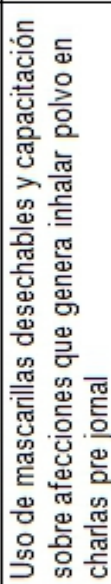 & 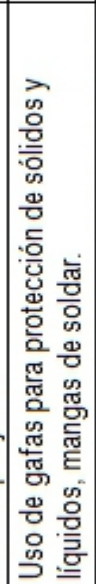 & 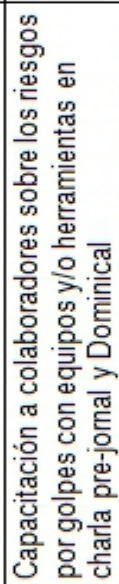 & 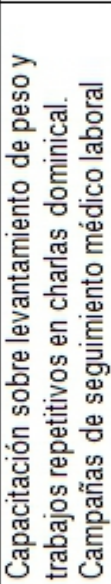 & 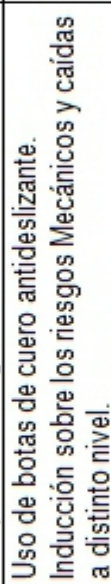 & 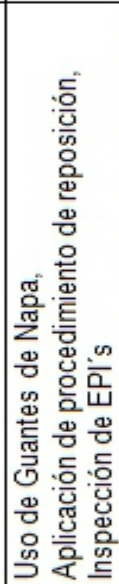 & 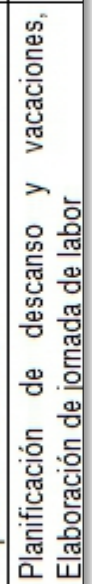 \\
\hline \multirow{7}{*}{$\begin{array}{l}\text { 嵒 } \\
\text { ơ } \\
\text { 点 } \\
\text { 엉 }\end{array}$} & - & $\times$ & $\times$ & $\times$ & $\times$ & $\times$ & $\times$ & $\times$ & $\times$ & $\times$ & \\
\hline & $\Sigma$ & & $\times$ & & & & & & & & \\
\hline & 4 & & & & & & & & & & $\times$ \\
\hline & 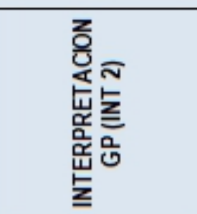 & 奀 & 季 & 喜 & 甭 & 蛹 & 甭 & 喜 & 甭 & 䇰 & 永 \\
\hline & פ' & 居 & 용 & 莒 & 䓌 & 足 & 总 & 总 & 高 & I & ㅇ \\
\hline & $d \exists$ & N & $\sim$ & $\sim$ & N & $\sim$ & $\sim$ & $\sim$ & $\sim$ & $\sim$ & $\sim$ \\
\hline & $\underset{\%}{\mathrm{NOIOISOdX} \exists}$ & 今. & 商 & 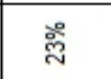 & 絗 & 商 & 商 & 商 & 商 & 今. & 商 \\
\hline
\end{tabular}

Fuente: Investigación de campo

Elaborado por: Ing. Ind. Mendoza Proaño Jairón Enrique 


\section{Metodología de evaluación de matriz de riesgo}

Cuadro 5. Valores para determinar el panorama de riesgo

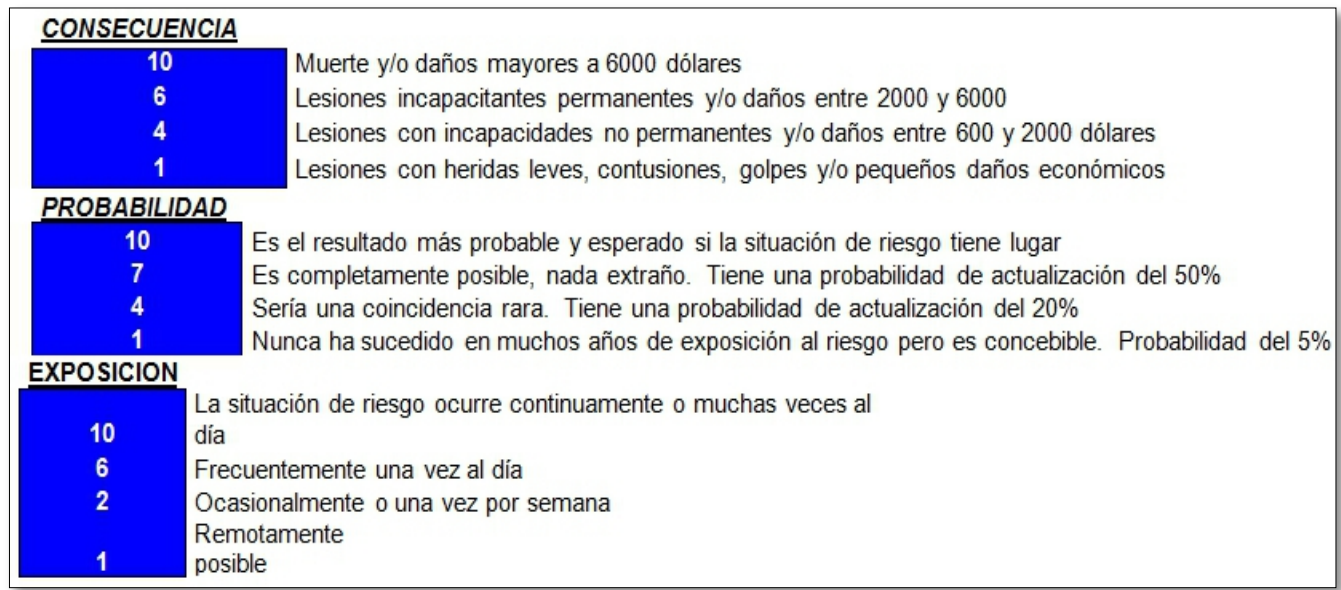

Fuente: Investigación de campo

Elaborado por: Mendoza Proaño Jairón Enrique

Cuadro 6. Leyendas

\begin{tabular}{|c|c|}
\hline \multicolumn{2}{|c|}{ LEYENDAS } \\
\hline $\mathbf{F}$ & FUENTE \\
\hline $\mathbf{M}$ & MEDIO \\
\hline $\mathbf{P}$ & PERSONA \\
\hline $\mathbf{C}$ & CONSECUENCIA \\
\hline $\mathbf{P}$ & PROBABILIDAD \\
\hline G.P. & GRADO DE PELIGROSIDAD \\
\hline Int.1 & INTERPRETACION GP \\
\hline \% Exp & PORCENTAJE DE EXPUESTO \\
\hline F.P. & FACTOR DE PONDERACION \\
\hline G.R. & GRADO DE REPERCUSIÒN \\
\hline Int.2 & INTERPRETACION GR \\
\hline
\end{tabular}

Fuente: Investigación de campo

Elaborado por: Mendoza Proaño Jairón Enrique

\section{Interpretación del grado de peligrosidad (gp)}

$\begin{array}{ll}\text { Bajo } & 1-300 \\ \text { Medio } & 301-600 \\ \text { Alto } & 6001-1000\end{array}$

$$
\% \text { expuestos }=\frac{\# \text { trabajadoresexpuestos }}{\# \text { totaldet rabajadores }} \times 100
$$

Interpretación del grado de repercusión (FP) 
Cuadro 7. Interpretación del grado de repercusión (FP)

\begin{tabular}{|c|c|}
\hline PORCENTAJE DE EXPUESTO & FACTOR DE PONDERANCION \\
\hline $1-20 \%$ & 1 \\
\hline $21-40 \%$ & 2 \\
\hline $41-60 \%$ & 3 \\
\hline $61-80 \%$ & 4 \\
\hline $81-100 \%$ & 5 \\
\hline
\end{tabular}

Fuente: Investigación de campo

Elaborado por: Mendoza Proaño Jairón Enrique

Interpretación del grado de repercusión (GR)

$\begin{array}{ll}\text { Bajo } & 1-1500 \\ \text { Medio } & 1501-3000 \\ \text { Alto } & 3001-5000\end{array}$

\section{$(\mathrm{GR})=\mathrm{GP} * \mathrm{FP}$}

\section{Esquema de un plan de intervención}

Cuadro 8. Esquema de un plan de intervención

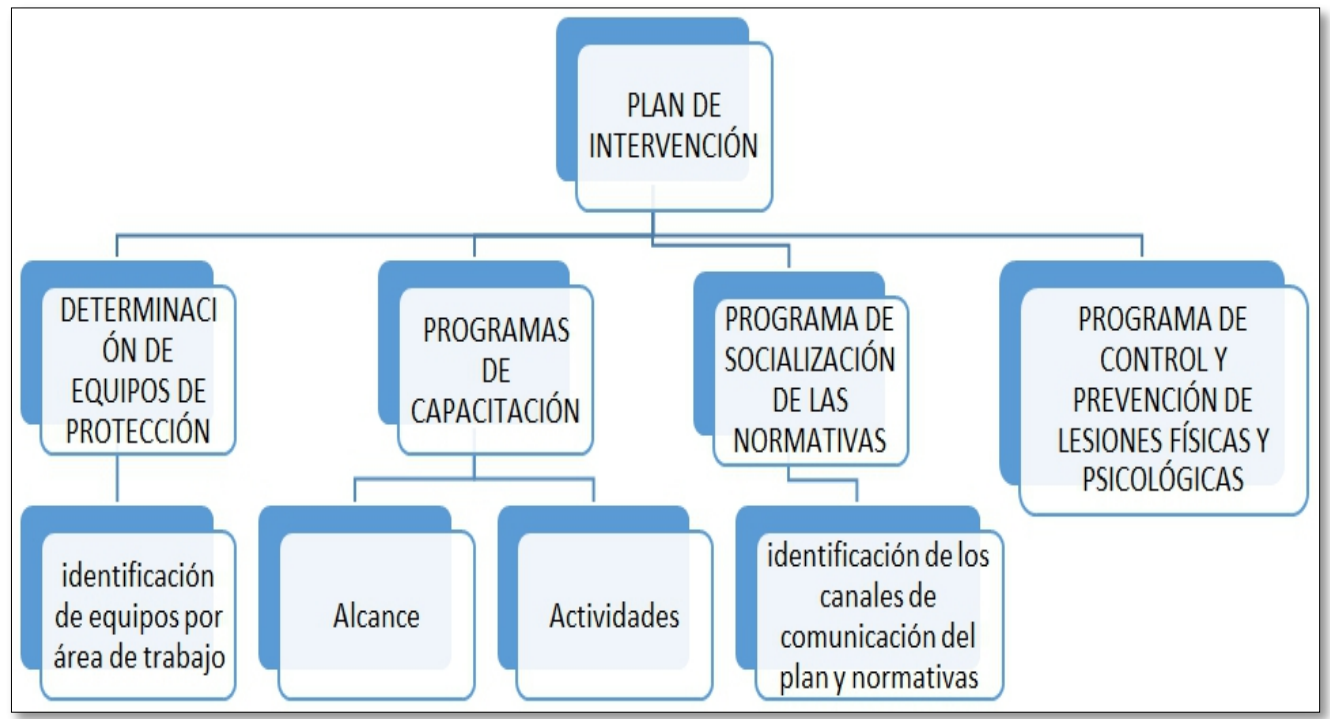

Fuente: Investigación de campo

Elaborado por: Mendoza Proaño Jairón Enrique

Bajo este esquema se deja establecida la necesidad de promover un estudio posterior para el diseño de cada una de las fases del Plan de Intervención tomando en consideración los resultados obtenidos bajo la metodología FINE para riesgos mecánicos en el área de fundición de estructuras metálicas de la Empresa Quintana Construcciones. 


\section{Conclusiones}

Como parte del análisis realizado a través de la metodología de William Fane, se logró identificar y evaluar los riesgos mecánicos al que son expuestos los trabajadores de la empresa Quintana Construcciones, específicamente en el área de fundición de estructuras metálicas, donde las diferentes actividades inmersas en la obra civil requiere del uso adecuado de equipos de protección, elemento necesario para garantizar la Salud y Seguridad Laboral.

De la misma forma, se comprobó que no existen procedimientos para el control preventivo de los equipos y herramientas de trabajo en las diversas, especialmente en las actividades relacionadas a los movimientos de tierra, donde la preparación de las maquinarias debe ser realizada de la mejor manera, así como su operatividad para evitar posibles deslizamientos del suelo.

Es preciso que en las instalaciones de trabajo se tome en consideración las debidas señalizaciones, ya que los resultados obtenidos de las encuestas determinan que la percepción de los trabajadores en relación a dichas normas de seguridad es casi nula, lo que puede generar un nivel de accidentabilidad progresivo, a la vez que limita el involucramiento del personal colaborativo en los procesos de seguridad laboral.

Los resultados obtenidos de la matriz determinan una serie de medidas de control actuales que deben ser corregidas, lo que da paso al diseño de un Plan de Intervención, donde se prevea las nuevas medidas a aplicar, su difusión, así como la puesta en marcha de las mismas para mitigar los riegos mecánicos en los trabajadores.

\section{References:}

1. Carbonel Slam, A., \& Torres Valle, A. (2011). Evaluación de percepción de riesgo ocupacional. Ingeniería Mecánica.

2. Cepyme (2011). Guía Técnica para la evaluación de riesgos y procedimientos de trabajo seguro conformado de metales, forja y fundición. Obtenido de Cepyme: http://www.conectapyme.com/documentacion/2011-FPRL3.pdf

3. Cepyme Aragón (2011). Manual de Seguridad y Prevención de Riesgos Laborales en Empresas del Sector Metal en obras de construcción. Obtenido de Fundación para la prevención de riesgos laborales:

http://www.conectapyme.com/documentacion/Prevencion/201205.pdf

4. Crisanto, T., \& Echeverría, I. (2015). estudio de factores de riesgos mecánicos presentes en accidentes laborales en una empresa metalmecánica. Obtenido de Universidad de las Fuerzas Armadas: 
http://www.ute.edu.ec/revistas/22/articulos/6c3f720a-e7ed-4a498f99-fd16537f8f80.pdf

5. Homero de Fundiciones (2013). Ficha Técnica de prevención de riesgos. Obtenido de Homero de Fundiciones: https://www.mutual.cl/portal/wcm/connect/9cc9e120-358d-4b97960f-397b59eb88d3/Hornero+de+fundiciones.pdf?MOD=AJPERES

6. Organización Internacional del Trabajo- OTI (2014). Organización Internacional del Trabajo. Obtenido de Seguridad y salud en el trabajo: http://www.ilo.org/global/topics/safety-and-health-at-work/ la ng--es/index.htm

7. Organización Mundial de la Salud (2013). Salud Ocupacional. Obtenido de Organización Mundial de la Salud: http://www.msal.gob.ar/index.php/home/salud-ocupacional

8. Rodríguez Garzón, I., Martínez Fiestas, M., \& López Alonso, M. (2012). El riesgo percibido por el trabajador de la construcción: ¿qué rol juega el oficio? revista de la construcción, 3 .

9. Sánchez Aguilar, M., Betzabé Pérez, G., \& González Díaz, G. (2011). Enfermedades potenciales derivadas de factores de riesgo presentes en la industria de producción de alimentos. revista de medicina y Seguridad del trabajo.

10. Universidad Carlos III de Madrid. (2014). Prevención de Riesgos Laborales. Obtenido de Universidad Carlos III de Madrid: http://portal.uc3m.es/portal/page/portal/laboratorios/prevencion_riesg os_laborales/manual/riesgos_mecanicos 


\section{Anexo1. Modelo de encuesta}

\section{Cuestionario Para La Identificación De Factores De Riesgos Mecánicos En Los Puestos De Trabajo}

OBJETIVO.- Estimado Colaborador (a), su opinión es muy importante para determinar la implementación de un Programa de intervención que reduzca los efectos de los riesgos mecánicos en la constructora PILEGGI. A continuación se presentará una serie de aspectos relevantes en este sentido, para que nos ayude a estimar los factores que inciden en la problemática, con la mayor objetividad posible, marcando con una equis (x) frente a cada opción que tenga mayor incidencia a su respuesta. Tomando en consideración las opciones SI, NO, N/S (no sabe) y N/P (no procede).

\begin{tabular}{|c|c|c|c|c|c|}
\hline $\mathbf{N}$ & OPCIONES & SI & NO & $\mathbf{N} / \mathbf{S}$ & $\mathbf{N} / \mathbf{P}$ \\
\hline & DISEÑO DEL PUESTO DE TRABAJO & & & & \\
\hline 1 & $\begin{array}{l}\text { Altura de la superficie de trabajo inadecuada para el tipo de } \\
\text { tarea o para las dimensiones del trabajador }\end{array}$ & & & & \\
\hline 2 & Espacio de trabajo insuficiente o inadecuado & & & & \\
\hline 3 & El diseño del puesto dificulta una postura de trabajo cómoda & & & & \\
\hline 4 & $\begin{array}{l}\text { Los controles y los indicadores asociados a su trabajo (mandos } \\
\text { de los equipos, tableros de instrumentación, etc.) se visualizan } \\
\text { con dificultad }\end{array}$ & & & & \\
\hline 5 & $\begin{array}{l}\text { Trabajo en situación de aislamiento o confinamiento (aunque } \\
\text { sea esporádicamente) }\end{array}$ & & & & \\
\hline 6 & $\begin{array}{l}\text { Zonas de trabajo y lugares de paso dificultados por exceso de } \\
\text { objetos }\end{array}$ & & & & \\
\hline \multirow[t]{2}{*}{7} & Carencia de vestuarios (si se precisan) & & & & \\
\hline & CONDICIONES AMBIENTALES & & & & \\
\hline 8 & $\begin{array}{l}\text { Temperatura inadecuada debido a la existencia de fuentes de } \\
\text { mucho calor o frío o a la inexistencia de un sistema de } \\
\text { climatización apropiado }\end{array}$ & & & & \\
\hline 9 & $\begin{array}{l}\text { Humedad ambiental inadecuada (ambiente seco o demasiado } \\
\text { húmedo) }\end{array}$ & & & & \\
\hline 10 & $\begin{array}{l}\text { Ruidos ambientales molestos o que provocan dificultad en la } \\
\text { concentración para la realización del trabajo }\end{array}$ & & & & \\
\hline 11 & $\begin{array}{l}\text { Insuficiente iluminación en su puesto de trabajo o entorno } \\
\text { laboral }\end{array}$ & & & & \\
\hline 12 & $\begin{array}{l}\text { Existen reflejos o deslumbramientos molestos en el puesto de } \\
\text { trabajo o su entorno }\end{array}$ & & & & \\
\hline 13 & Percibe molestias frecuentes en los ojos & & & & \\
\hline 14 & $\begin{array}{l}\text { Molestias frecuentes atribuibles a la calidad del medio ambiente } \\
\text { interior (aire viciado, malos olores, polvo en suspensión, } \\
\text { productos de limpieza, etc.) }\end{array}$ & & & & \\
\hline
\end{tabular}




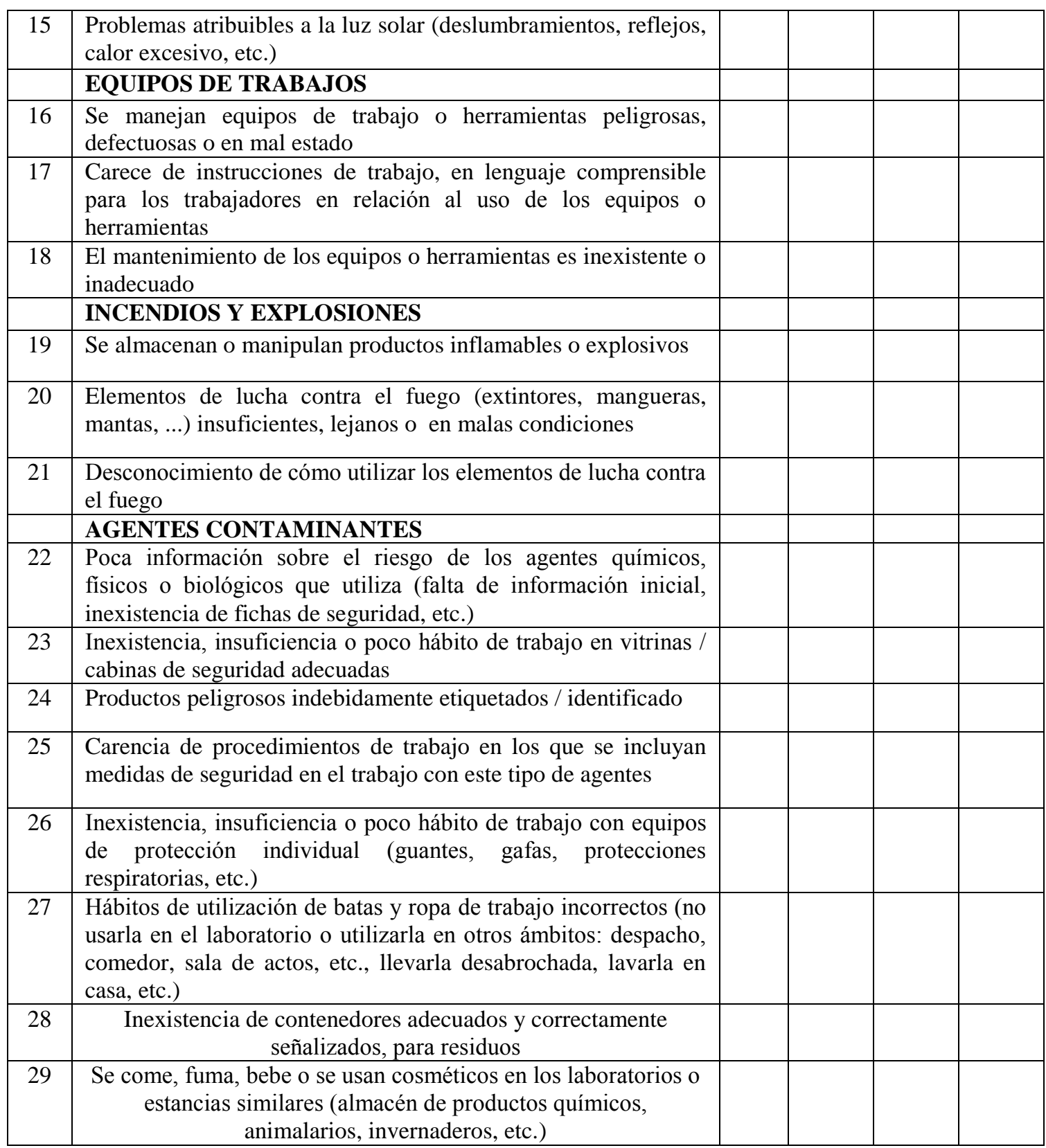

Fuente: Investigación de campo

Elaborado por: Mendoza Proaño Jairón Enrique 